\title{
ON A CLASS OF NON-SELF-ADJOINT DIFFERENTIAL OPERATORS
}

\author{
R. R. D. KEMP
}

The problem of spectral analysis of non-self-adjoint (and non-normal) operators has received considerable attention recently. Livsic (5), and more recently Brodskii and Livsic (1) have considered operators on Hilbert space with completely continuous imaginary parts. Dunford (3) has generalized the notion of spectral measure and defined a class of spectral operators on Hilbert and Banach space. Schwartz (8) and Rota (7) have investigated conditions under which a differential operator will be spectral. The work of Naimark (6) and the author (4) on non-self-adjoint differential operators leads to an expansion theorem which implicitly defines a type of spectral measure. However the projections involved in this will not in general be bounded, much less uniformly bounded.

The present paper is a generalization of $(4)$ to $n$th order differential operators. If $p(\mu)=\mu^{n}+a_{1} \mu^{n-1}+a_{2} \mu^{n-2}+\ldots+a_{n}$ is a polynomial with complex coefficients, the differential expression $p(-i D)$, where $D=d / d x$, can be used to define a closed operator on $L^{p}(-\infty, \infty)$ for $1 \leqslant p \leqslant \infty$. On $L^{2}(-\infty, \infty)$ it is a normal operator, and its spectrum $\{\lambda \mid \lambda=p(t)$ for real $t\}$ is the same in all these spaces. We shall consider operators $L$ arising from this operator $L_{0}$ by the addition of a linear differential operator of order $n-2$ with coefficients which are suitably small at $\pm \infty$. The previous paper (4) dealt with the simplest case $n=2, a_{1}=a_{2}=0$.

We shall analyse the spectrum of $L$ and show that it is determined by $p(\mu)$ except for a bounded set of characteristic values which are the zeros of certain analytic functions. We shall also obtain an expansion of the Green's function, and from this an expansion in characteristic functions for a suitably restricted class of functions.

1. Solutions of $L y=\lambda y$. Now $L=L_{0}+L_{1}$ where $L_{1}=\sum_{j=2}^{n} b_{j}(x)$ $(-i D)^{n-j}$. We assume that $\left(x^{2}+1\right)^{r} b_{j}(x) \in L^{1}(-\infty, \infty)$ for a suitable $r$. This $r$ is the multiplicity of the root of $p^{\prime}(\mu)=0$ which has highest multiplicity.

The equation $L y-\lambda y=f$ is equivalent to a system of $n$ first order equations

$$
Y^{\prime}=[A+B] Y+F
$$

Received June 22, 1959. 
where $Y$ and $F$ are $n \times 1$ matrices with entries $y_{1}, y_{2}, \ldots, y_{n}$ and $0,0, \ldots$, $0, i^{n} f$ respectively, and $A$ and $B$ are $n \times n$ matrices with entries

$$
a_{j k}=\delta_{j k-1}-\delta_{j n} i^{n-k+1} a_{n-k+1}+\delta_{j n} \delta_{k 1} i^{n} \lambda \text { and } b_{j k}=-\delta_{j n} i^{n-k+1} b_{n-k+1}(x)
$$

respectively, with the convention that $b_{1}(x) \equiv 0$.

In order to obtain the Green's function for $L y-\lambda y=f$ we first construct certain solutions of (1.1) for $F=0$, and use them to construct the Green's matrix for (1.1). We note that if $\lambda$ is such that $p(\mu)=\lambda$ has $n$ distinct solutions $\mu_{1}, \mu_{2}, \ldots, \mu_{n}$, then $Z^{\prime}=A Z$ has a fundamental matrix $M \exp [i \theta x]$ where $\theta=\left[i \mu_{j} \delta_{j k}\right]$ and $M=\left[\left(i \mu_{j}\right)^{k-1}\right]$. Using this we obtain an integral equation equivalent to (1.1) with $F=0$ in the form

$$
Y(x)=M \exp [i \theta x] c_{0}+\int^{x} M \exp [i \theta(x-\xi)] M^{-1} B(\xi) Y(\xi) d \xi,
$$

where $c_{0}$ is a constant $n \times 1$ matrix and the lower limits on the integrals (each element in the column matrix) are arbitrary.

We shall obtain solutions of (1.2) which are asymptotic to solutions of $Z^{\prime}=A Z$, but before stating this result we must make some additional remarks. If $\lambda_{1}$ is such that $p(\mu)=\lambda_{1}$ has $n$ distinct solutions then the same is true for $\lambda$ sufficiently close to $\lambda_{1}$, and the $n$ solutions $\mu_{1}(\lambda), \mu_{2}(\lambda), \ldots, \mu_{n}(\lambda)$ of $p(\mu)=\lambda$ are analytic functions of $\lambda$ in this neighbourhood of $\lambda_{1}$. These functions have branch points at $\lambda_{j}{ }^{0}=p\left(\mu_{j}{ }^{0}\right) j=1,2, \ldots, n-1$ where $\mu_{j}{ }^{0} j=1,2, \ldots$, $n-1$ are the $n-1$ solutions of $p^{\prime}(\mu)=0$. In any simply connected region containing no $\lambda_{j}{ }^{0}$ the functions $\mu_{k}(\lambda)$ are analytic. In solving (1.2) the curves $\gamma_{j k}$ defined by the equations $\operatorname{Im} \mu_{\jmath}=\operatorname{Im} \mu_{k}$ will be important. We shall first solve (1.2) in regions $D$ which are simply connected, bounded away from branch points, and contain none of the curves $\gamma_{\jmath k}$ in their interiors.

Theorem 1.1. There are solutions $\phi_{1}, \phi_{2}, \ldots, \phi_{n}$ and $\tilde{\phi}_{1}, \tilde{\phi}_{2}, \ldots, \tilde{\phi}_{n}$ of $(1.2)$ which exist for all bounded $\lambda$ in $\bar{D}$. The matrices $\Phi=\left[\phi_{1} \phi_{2} \ldots \phi_{n}\right]$ and $\tilde{\Phi}=$ $\left[\tilde{\phi}_{1}, \tilde{\phi}_{2} \ldots \tilde{\phi}_{n}\right]$ are analytic in $\lambda(\lambda \in D)$ for fixed $x$, and have the following asymptotic behaviour:

$$
\begin{aligned}
& \Phi(x, \lambda)=M \exp [i \theta x](I+o(1)) x \rightarrow \infty, \\
& \tilde{\Phi}(x, \lambda)=M \exp [i \theta x](I+o(1)) x \rightarrow-\infty,
\end{aligned}
$$

where $I=\left[\delta_{j k}\right]$ is the identity matrix.

This theorem is a modification of Theorem 8.1 in Coddington and Levinson (2; p. 92), and its proof will be omitted. We shall need to know the relation that $\Phi_{1}(x, \lambda)$ and $\tilde{\Phi}_{1}(x, \lambda)$ in $D_{1}$ bear to the corresponding matrices in $D_{2}$ where $\bar{D}_{1} \cap \bar{D}_{2}$ is a portion of one (or more) of the curves $\gamma_{j k}$. By an examination of the particular cases of (1.2) used in proving Theorem 1.1 one can see that $\phi_{p 1}(x, \lambda)=\phi_{p 2}(x, \lambda)$ on $\gamma_{j k}$ unless $p=j$ or $p=k$, and that $\phi_{j 1}(x, \lambda)=$ ${ }_{j 2}(\phi x, \lambda)+c_{k} \phi_{k 2}(x, \lambda)+$ a linear combination of terms $\phi_{p 2}(x, \lambda)$ which are of 
lower order of growth at $\infty$ than $\phi_{j}$ or $\phi_{k}$. A similar relation holds for $\phi_{k}$ and completely analogous results are true for the $\tilde{\phi}_{p}$ 's. The generalization to the case where several of the curves $\gamma_{j k}$ coincide is immediate.

We shall also be interested in the asymptotic behaviour of $\Phi$ and $\tilde{\Phi}$ as $|\lambda| \rightarrow \infty$. We see very easily that for large $|\lambda|$ the $\mu_{j}$ 's can be renumbered so that $\mu_{j}=\alpha_{j} \lambda^{1 / n}\left(1+0\left(|\lambda|^{-1 / n}\right)\right)$ where $0 \leqslant \arg \lambda^{1 / n}<(2 \pi) / n$ and $\alpha_{j}=\exp (2 \pi j i / n)$. Since we are bounded away from the branch points it is easy to show by direct calculation that each entry in $M^{-1} B(x) M$ is less than or equal to $K|\lambda|^{-1 / n} \sum_{j=2}^{n}\left|b_{j}(x)\right|$. From this we obtain

$$
\Phi(x, \lambda)=M \exp [i \theta x]\left(I+0\left(|\lambda|^{-1 / n}\right)\right) \text { as }|\lambda| \rightarrow \infty,
$$

and

$$
\tilde{\Phi}(x, \lambda)=M \exp [i \theta x]\left(I+0\left(|\lambda|^{-1 / n}\right)\right) \text { as }|\lambda| \rightarrow \infty,
$$

provided $|\lambda| \rightarrow \infty$ while $\lambda \in D$. It is not hard to see from the asymptotic behaviour of the $\mu_{j}$ 's that such regions $D$ do exist.

If $D$ is included in another such region $D_{1}$, which extends closer to the branch points, the solutions making up $\Phi$ and $\tilde{\Phi}$ are changed, but $\Phi_{1}=\Phi C$ and $\tilde{\Phi}_{1}=\tilde{\Phi} C^{\prime}$ where $C$ and $C^{\prime}$ are constant matrices. It is easy to use (1.3) and (1.4) to conclude that $C$ has units along the main diagonal and zeros below and that $C^{\prime}$ is the transpose of such a matrix, provided that the $\mu_{j}$ 's are numbered so that $\operatorname{Im} \mu_{1}>\operatorname{Im} \mu_{2}>\ldots>\operatorname{Im} \mu_{n}$.

Finally we shall also need solutions of (1.1) when $\lambda$ is a branch point of the functions $\mu_{j}(\lambda)$. At such a point the $\mu_{j}$ 's coincide in groups and the solutions of $p(\mu)=\lambda$ will be denoted by $\mu_{1}, \mu_{2}, \ldots, \mu_{r}$ with multiplicities $m_{1}, m_{2}, \ldots$, $m_{r}\left(\sum_{j=1}^{r} m_{j}=n\right)$. A fundamental matrix of $Z^{\prime}=A Z$ will then have the more complicated form $M_{1} D \exp \left[i \theta_{1} x\right]$ where $\theta_{1}$ is a diagonal matrix with $m_{1} \mu_{1}$ 's, then $m_{2} \mu_{2}$ 's, etc. down the diagonal. The matrix $D$ can be partitioned so that it has zero matrices off the diagonal and blocks $D_{1}, D_{2}, \ldots, D_{r}$ down the diagonal. $D_{k}$ is $m_{k} \times m_{k}$, has zeros below the main diagonal, and $\left(D_{k}\right)_{p q}=$ $x^{q-p} /(q-p)$ ! for $q \geqslant p$. Thus $D^{-1}(x)=D(-x)$ and $D$ commutes with $\exp \left[i \theta_{1} x\right]$. The columns of $M_{1}$ are determined in $r$ groups of $m_{1}, \ldots, m_{r}$ columns. The first column in the $k$ th group is a $n \times 1$ matrix satisfying the equation $\left(A-i \mu_{k}\right) z_{1}{ }^{(k)}=0$, and the $j$ th column in this group is a solution of the equation $\left(A-i \mu_{k}\right) z_{j}{ }^{(k)}=z_{j-1}{ }^{(k)}\left(j=2, \ldots, m_{k}\right)$. In particular, a suitable choice of constants leads to the following formula for the $l$ th entry in $z_{j}^{(k)}$ :

$$
\left(z_{j}^{(k)}\right)_{l}=\left\{\begin{array}{cl}
\frac{(l-1) !}{(j-1) !(l-j) !}\left(i \mu_{k}\right)^{l-j} & l \geqslant j \\
0 & l<j .
\end{array}\right.
$$

Thus the equation corresponding to (1.2) is

$$
\begin{array}{r}
Y(x)=M_{1} D(x) \exp \left[i \theta_{1} x\right] c_{0}+\int^{x} M_{1} D(x-\xi) \exp \left[i \theta_{1}(x-\xi)\right] \\
M_{1}^{-1} B(\xi) Y(\xi) d \xi .
\end{array}
$$


THEOREM 1.2. There exist solutions $\phi_{k}^{(j)}(x)$ and $\tilde{\phi}_{k}^{(j)}(x)$ of (1.7) for $j=1, \ldots, r ; k=1, \ldots, m_{j}$ such that

$$
\phi_{k}^{(j)}(x)=\frac{x^{k-1}}{(k-1) !} e^{i \mu_{j} x}\left(z_{1}^{(j)}+o(1)\right) \quad \text { as } \quad x \rightarrow \infty .
$$

The solution $\tilde{\phi}_{k}^{(j)}(x)$ has the same asymptotic behaviour as $x \rightarrow-\infty$.

The proof of this theorem is a modification of that of Theorem 1.1, and will be omitted. The appropriate modification is described in Problem 35 of Coddington and Levinson (2, p. 106). We might note that it is at this point that the full strength of the assumptions on $b_{j}(x)$ are used (in Theorem 1.1 it is only necessary to assume $\left.b_{j}(x) \in L^{1}\right)$.

2. Construction of the Green's function. We shall now discuss the solution of (1.1) for $F \neq 0$ when the equation $p(\mu)=\lambda$ has no real solutions. For any matrix or vector function we shall use the following notations:

$$
|A(x)|^{p}=\sum_{i, j}\left|A_{i j}(x)\right|^{p} \text {, with }|A(x)|=|A(x)|^{1}
$$

and

$$
\|A\|_{p}=\left\{\int_{-\infty}^{\infty}|A(x)|^{p} d x\right\}^{1 / p} .
$$

As $p(\mu)=\lambda$ has no real solutions we can number the solutions $\mu_{1}, \mu_{2}, \ldots, \mu_{n}$ so that $\operatorname{Im} \mu_{1} \geqslant \operatorname{Im} \mu_{2} \geqslant \ldots \geqslant \operatorname{Im} \mu_{m}>0>\operatorname{Im} \mu_{m+1} \geqslant \operatorname{Im} \mu_{m+2} \geqslant \ldots \geqslant$ Im $\mu_{n}$. Thus $\phi_{1}, \phi_{2}, \ldots, \phi_{m}$ are exponentially small at $\infty$ and $\tilde{\phi}_{m+1}, \tilde{\phi}_{m+2}, \ldots$, $\tilde{\phi}_{n}$ are exponentially small at $-\infty$. We shall partition our matrices as follows:

$$
\Phi=\left[\begin{array}{ll}
\Phi_{11} & \Phi_{12} \\
\Phi_{21} & \Phi_{22}
\end{array}\right], \quad \tilde{\Phi}=\left[\begin{array}{ll}
\tilde{\Phi}_{11} & \tilde{\Phi}_{12} \\
\tilde{\Phi}_{21} & \tilde{\Phi}_{22}
\end{array}\right],
$$

where $\Phi_{11}$ and $\tilde{\Phi}_{11}$ are $m \times m$ and the rest coherent with this. We now define

$$
\Psi=\left[\begin{array}{ll}
\Phi_{11} & \tilde{\Phi}_{12} \\
\Phi_{21} & \tilde{\Phi}_{22}
\end{array}\right]
$$

and provided that $\Psi^{-1}$ exists

$$
K(x, \xi, \lambda)= \begin{cases}{\left[\begin{array}{cc}
\Phi_{11}(x) & 0 \\
\Phi_{21}(x) & 0
\end{array}\right] \Psi^{-1}(\xi)} & \xi \leqslant x \\
-\left[\begin{array}{cc}
0 & \tilde{\Phi}_{12}(x) \\
0 & \tilde{\Phi}_{22}(x)
\end{array}\right] \Psi^{-1}(\xi) & x \leqslant \xi .\end{cases}
$$

THEOREM 2.1. If $\Psi$ is non-singular for a particular value of $\lambda$ then $K(x, \xi, \lambda)$ is the Green's matrix for the solution of (1.1).

Proof. By this we mean that if $F$ is a vector function with $\|F\|_{p}<\infty$ (that is, $F \in L^{p}$ ) then the vector function 


$$
\hat{y}(x)=\int_{-\infty}^{\infty} K(x, \xi, \lambda) F(\xi) d \xi
$$

is the unique solution of (1.1) which belongs to $L^{p}$.

If two such solutions exist then there is a solution of (1.1) with $F=0$ which belongs to $L^{p}$. As this cannot be exponentially large at either $+\infty$ or $-\infty$ it is a linear combination of $\phi_{1}, \phi_{2}, \ldots, \phi_{m}$; and also a linear combination of $\tilde{\phi}_{m+1}, \tilde{\phi}_{m+2}, \ldots, \tilde{\phi}_{n}$. A non-zero solution cannot have this property if $\Psi$ is non-singular, so an $L^{p}$ solution of (1.2) must be unique if it exists.

An examination of the definition of $K(x, \xi, \lambda)$ yields the fact that if the integral in (2.1) exists it must be differentiable and satisfy (1.1). Thus the burden of proof is in showing that $\hat{y}$ defined by (2.1) exists and belongs to $L^{p}$.

Since $\Phi$ and $\tilde{\Phi}$ are both fundamental matrices $\Phi=\tilde{\Phi} C$ where $C$ is a constant matrix. We partition $C, C^{-1}, \Phi^{-1}, \tilde{\Phi}^{-1}$ in the same way as $\Phi$ and $\tilde{\Phi}$, and use the notations $\Phi^{-1}=\left[\Phi^{i j}\right], \tilde{\Phi}^{-1}=\left[\tilde{\Phi}^{i j}\right], C=\left[C_{i j}\right]$, and $C^{-1}=\left[C^{i j}\right]$. We also note that if $\lambda$ is not a branch point $\Phi$ and $\tilde{\Phi}$ behave asymptotically like $M \exp [i \theta x]$ so their inverses behave asymptotically like $\exp [-i \theta x] M^{-1}$. Although such a precise statement cannot be made if $\lambda$ is a branch point, bounds on the elements in $\Phi^{-1}$ and $\tilde{\Phi}^{-1}$ can be obtained.

For $x$ and $\xi$ non-negative we write $K(x, \xi, \lambda)$ in the form

$$
K(x, \xi, \lambda)= \begin{cases}\Phi(x)\left[\begin{array}{rr}
I & -A \\
0 & 0
\end{array}\right] \Phi^{-1}(\xi), & \xi \leqslant x, \\
-\Phi(x)\left[\begin{array}{rr}
0 & A \\
0 & I
\end{array}\right] \Phi^{-1}(\xi), & x \leqslant \xi,\end{cases}
$$

where $A=C^{12}\left(C^{22}\right)^{-1}$. A careful examination of this yields the fact that, whether $\lambda$ is a branch point or not, each element is bounded by $K \exp [-\delta|x-\xi|]$ where $\delta$ and $K$ are positive constants and $\delta<\min \left[\left|\operatorname{Im} \mu_{m}\right|,\left|\operatorname{Im} \mu_{m+1}\right|\right]$. This latter condition allows for bounds on the terms which are of order $x^{k} e^{i_{\mu_{m}} x}$ for some $k$.

If $x \leqslant 0 \leqslant \xi$ we rewrite $K(x, \xi, \lambda)$ in the form

$$
K(x, \xi, \lambda)=-\tilde{\Phi}(x)\left|\begin{array}{rr}
0 & 0 \\
0 & C_{22}^{-1}
\end{array}\right| \Phi^{-1}(\xi)
$$

and again find that each element is bounded by $K \exp [-\delta|x-\xi|]$.

On performing similar analyses for $x, \xi$ non-positive and for $x \geqslant 0 \geqslant \xi$ we obtain

$$
|K(x, \xi, \lambda)| \leqslant K \exp [-\delta|x-\xi|]
$$

where $\delta$ and $K$ are as before although $K$ may have been increased.

Thus, from (2.1)

$$
\begin{aligned}
|y(x)| & \leqslant K \int_{-\infty}^{\infty} e^{-\delta|x-\xi|}|F(\xi)| d \xi \\
& \leqslant K\left\{\int_{-\infty}^{\infty} e^{-p \delta|x-\xi| / 2}|F(\xi)|^{p} d \xi\right\}^{1 / p}\left\{\int_{-\infty}^{\infty} e^{-q \delta|x-\xi| / 2} d \xi\right\}^{1 / q}
\end{aligned}
$$


by Hölder's inequality. Thus, using a change of variables and the Fubini theorem we obtain

$$
\begin{aligned}
\int_{-\infty}^{\infty}|y(x)|^{p} d x & \leqslant K^{p}(4 / q \delta)^{p / q} \int_{-\infty}^{\infty} \int_{-\infty}^{\infty} e^{-p \delta|\xi| / 2}|F(x-\xi)|^{p} d \xi d x \\
& \leqslant K^{p}(4 / q \delta)^{p / q} \int_{-\infty}^{\infty} e^{-p \delta|\xi| / 2}\left\{\int_{-\infty}^{\infty}|F(x)|^{p} d x\right\} d \xi \\
& \leqslant K_{1}|| F||_{p}^{p} .
\end{aligned}
$$

Thus $y(x)$ exists and belongs to $L^{p}$ for all $p \geqslant 1$.

COROLlary 2.1. The Green's function for the differential operator $L$ is $G(x, \xi, \lambda)=i^{n} K_{1 n}(x, \xi, \lambda)$ where $K_{1 n}(x, \xi, \lambda)$ is the element in the first row and $n$th column of $K(x, \xi, \lambda)$, provided that $K(x, \xi, \lambda)$ exists.

We might note that $K(x, \xi, \lambda)$, and thus $G(x, \xi, \lambda)$ may very well exist even if $p(\mu)=\lambda$ has real solutions, although they have not been proved to be Green's functions in this case.

Since the theorem depends upon $\Psi$ being non-singular we must examine this question in detail. Note that for real $t$ the equation $\lambda=p(t)$ defines a curve in the complex plane, which will in general split the complex plane up into several regions $D_{j}(j=1,2, \ldots, p)$. Suppose $\lambda_{0}$ is in one of these regions and does not lie on any $\gamma_{j k}$. Then in a neighbourhood of $\lambda_{0}$ no $\operatorname{Im} \mu_{j}$ changes sign. Thus in this neighbourhood $m$ is fixed and $K(x, \xi, \lambda)$ is analytic in $\lambda$. In crossing a $\gamma_{j k}$ while remaining within $D_{j}$ the $\phi_{j}$ 's and $\tilde{\phi}_{j}$ 's may change, but from the considerations of $\$ 1$ we see that if on one side $\operatorname{Im} \mu_{1}>\operatorname{Im} \mu_{2}>\ldots>\operatorname{Im} \mu_{n}$ then on $\gamma_{j k}$ the $\Phi$ matrix from that side, $\Phi_{1}$, will have the asymptotic form.

$$
\Phi_{1}=M \exp [i \theta x] A_{1}[I+o(1)] \text { as } x \rightarrow \infty
$$

where $A_{1}$ has units along the main diagonal and zeros below it. From the other side the only difference will be that the order of the $\mu_{j}$ 's has been altered. Thus

$$
\Phi_{2}=M \exp [i \theta x] Q A_{2}[I+o(1)] \text { as } x \rightarrow \infty
$$

where $A_{2}$ is of the same form as $A_{1}$ and $Q$ rearranges the columns of $M \exp [i \theta x]$ appropriately. Hence $\Phi_{1}=\Phi_{2} A_{2}{ }^{-1} Q^{-1} A_{1}$. Similarly $\tilde{\Phi}_{1}=\tilde{\Phi}_{2} B_{2}{ }^{-1} Q^{-1} B_{1}$ where $B_{1}$ and $B_{2}$ have units along the main diagonal and zeros above. Note that $A_{2}{ }^{-1}$ and $B_{2}{ }^{-1}$ have the same form as $A_{2}$ and $B_{2}$ respectively and that it is impossible to have a $\gamma_{j k}$ here where $j \leqslant m<k$ or $k \leqslant m<j$. This implies that in $Q=\left[Q_{i j}\right]$, partitioned as before, we have $Q_{12}=Q_{21}=0$. Using this and the definition of $\Psi$ we see that

$$
\Psi_{1}=\Psi_{2}\left[\begin{array}{lr}
P_{11} & 0 \\
0 & P_{22}
\end{array}\right]
$$

where $P_{11}$ and $P_{22}$ have determinant \pm 1 (as $A_{j}, B_{j}$, and $Q$ have this property).

It should be noted that a particular set of solutions $\phi_{j}$ or $\tilde{\phi}_{j}$ exist in a set which is bounded away from the branch points. Thus as well as noting the 
behaviour of $\Psi$ and finally of $K(x, \xi, \lambda)$ across the curves $\gamma_{j k}$, we must consider how they are affected by a replacement of the $\phi_{j}$ 's and $\tilde{\phi}_{j}$ 's by a different set which exist in a larger region extending closer to the branch points. An examination of the changes required in the particular cases of (1.2) to obtain such a new set of solutions shows that in their common domain the two $\Psi$ 's will again be related by an equation of the form of (2.3) with $P_{11}$ having units on the main diagonal and zeros below, and $P_{22}$ being the transpose of such a matrix.

These remarks may be summarized in the following theorem.

THEOREM 2.2. If the branch points are removed from the regions $D_{j}$ the matrix $K(x, \xi, \lambda)$ is analytic in the remaining portion except at points where $\Psi$ is singular. If we define a function $W_{j}(\lambda)= \pm(\operatorname{det} \Psi) \exp \left(i a_{1} x\right]$, by fixing the sign at some point and then choosing coherent signs on the two sides of each $\gamma_{j k}$ we obtain a function locally analytic in $D_{j}$ except at branch points, which may be double-valued. [Note that $W_{j}(\lambda)$ is independent of $x$ ].

Proof. The first statement is obvious from (2.3). If $D_{j}$ contains no branch points the functions $\mu_{1}(\lambda), \mu_{2}(\lambda), \ldots, \mu_{n}(\lambda)$ are everywhere distinct and analytic throughout. Thus $W_{j}(\lambda)$ is also analytic throughout, but if $D_{j}$ contains a branch point continuation of $\mu_{1}(\lambda), \ldots, \mu_{n}(\lambda)$ around a curve surrounding it it will result in returning to $\mu_{\pi(1)}(\lambda), \ldots, \mu_{\pi(n)}(\lambda)$ where $\pi$ is a permutation of the integers $1,2, \ldots, n$ which is not the identity. This may result in a change of the sign of $W_{j}(\lambda)$, in which case $W_{j}(\lambda)$ is double-valued in $D_{j}$.

Thus the points where $\Psi$ is singular in any such $D_{j}$ are determined by the zeros of a (perhaps double-valued) function locally analytic except at branch points. Thus unless this function is identically zero the points where $\Psi$ is singular form a countable set in $D_{j}$ with limit points (if any) at the branch points and on the boundary. We will attempt to characterize this set somewhat more completely, and to characterize the regions $D_{j}$ where $W_{j}(\lambda)$ can be identically zero. Such $D_{j}$ 's will be called exceptional.

On investigating the region of validity of the original definition of $W_{j}(\lambda)$ by a determinant we see that it is valid across an arc of $\lambda=p(t)$ which does not coincide with a $\gamma_{k l}\left(=\gamma_{l k}\right)$ with $k \leqslant m<l$. It is easily seen that such a $\gamma_{k l}$ would have to be $\gamma_{m m+1}$ so that this portion of $\lambda=p(t)$ is traversed twice. Thus the original definition of $W_{j}(\lambda)$ is valid across an arc of $\lambda=p(t)$ which bounds $D_{j}$ and contains no double points. Hence if $W_{j}(\lambda) \neq 0$ the limit points of its zeros can only lie at branch points in $D_{j}$, boundary points of $D_{j}$ which are multiple points of the curve $\lambda=p(t)$, or outside of $D_{j}$. One notes that the original definition of $W_{j}(\lambda)$ is valid outside of $D_{j}$ up to $\gamma_{k l}$ with $k \leqslant m<l$ (using (2.3) to cross other $\gamma_{p q}$ ) and if this results in it being valid in a neighbourhood of some arc extending to $\infty$ we may use (1.5) and (1.6) to conclude that in this neighbourhood

$$
\begin{aligned}
W_{j}(\lambda) & = \pm e^{i a_{1} x} \operatorname{det}\left\{M \exp [i \theta x]\left(I+O\left(|\lambda|^{-1 / n}\right)\right)\right\} \\
& = \pm(\operatorname{det} M)\left(1+O\left(|\lambda|^{-1 / n}\right)\right) \text { as }|\lambda| \rightarrow \infty
\end{aligned}
$$


For large $|\lambda|$ we again use the asymptotic behaviour of the appropriately numbered $\mu_{j}$ 's to find that

$$
\operatorname{det}(M)= \pm n(-\lambda)^{\frac{1}{2} n-\frac{1}{2}} 2^{\frac{1}{2}(n-1)(n-2)}\left[1+O\left(|\lambda|^{-1 / n}\right)\right] \text { as }|\lambda| \rightarrow \infty .
$$

Thus $W_{j}(\lambda)$, which can be so continued, cannot be identically zero. This proves the following theorem.

Theorem 2.3. In order to be exceptional, $D_{j}$ must be bounded from $\infty$ by curves $\gamma_{k l}$ with $k \leqslant m<l$. In any non-exceptional $D_{j}$ (or an exceptional $D_{j}$ where $W_{j}(\lambda)$ is not identically zero), the points where $\Psi$ is singular make up a discrete bounded set with limit points at the branch points, or at points $\lambda_{0}$ on the boundary where $p(\mu)=\lambda_{0}$ has more than one real root.

We might note that there is always at least one unbounded $D_{j}$, which must therefore be non-exceptional, and that exceptional $D_{j}$ 's can exist, for example, if

$$
p(\mu)=\left[\frac{27}{4} \mu^{2}\left(\mu^{2}-1\right)^{2}-1\right]\left[\frac{27}{4} \mu^{2}\left(\mu^{2}-1\right)^{2}+\frac{3 \sqrt{3}}{2} i \mu\left(\mu^{2}-1\right)-1\right]
$$

it is found that $\lambda=u+i v=p(t)$ is the curve $u=\left(\tau^{2}-1\right)^{2}, v=\tau\left(\tau^{2}-1\right)$, and that the loop in it between $\tau=-1$ and $\tau=+1$ is traced three times (so encloses an exceptional region).

It is perhaps also worthwhile to remark that this classification of the regions $D_{j}$, and the characterization of the possible limit points of the zeros of $W_{j}(\lambda)$ depends only upon $L_{0}=\mathrm{p}(-i D)$, and not on the perturbing operator $L_{1}$.

3. The spectrum of the operators $L$ and $L^{*}$. We have seen above that there are values of $\lambda$ for which the differential equation $L y-\lambda y=f$ possesses a Green's function $G(x, \xi, \lambda)$ which generates a bounded operator on $L^{p}(-\infty, \infty)$ for $1 \leqslant p \leqslant \infty$. Fix such a value $\lambda_{0}$.

Theorem 3.1. The operator $L=L_{0}+L_{1}$ with domain $D_{L, p}=\left\{y \in L^{p} \cap\right.$ $C^{n-1} \mid y^{(n-1)}$ is absolutely continuous and $\left.L y \in L^{p}\right\}$, is a closed operator on $L^{p}(-\infty, \infty)$.

Proof. Let $y_{n} \in D_{L, p}, y_{n} \rightarrow y_{0}$ and $L y_{n} \rightarrow f$. Now

$$
y_{n}(x)=\int_{-\infty}^{\infty} G\left(x, \xi, \lambda_{0}\right)\left(L-\lambda_{0}\right) y_{n}(\xi) d \xi,
$$

and the boundedness of the operator generated by $G$ implies that

$$
y_{0}(x)=\int_{-\infty}^{\infty} G\left(x, \xi, \lambda_{0}\right)\left[f(\xi)-\lambda_{0} y_{0}(\xi)\right] d \xi .
$$

From this equation it follows immediately that $y_{0} \in D_{L, P}$ and $L y_{0}=f$.

We shall now introduce the adjoint operator $\widetilde{L}$ on $L^{q}(-\infty, \infty)$. Here $(1 / p+1 / q=1)$. If $p=1, q=\infty$ and if $p=\infty$ we shall introduce an adjoint on $L^{1}(-\infty, \infty)$ although this is not the dual space. Let $\widetilde{L}_{k}=i D \widetilde{L}_{k-1}+$ $a_{k}+b_{k}(x)$ where $\widetilde{L}_{0}=1$ and $k=0,1, \ldots, n$, and define the operator $\widetilde{L}$ with 
domain $D_{\tilde{L}, p}=\left\{y \in L^{p} \mid \tilde{L}_{k} y\right.$ is absolutely continuous $k=0,1, \ldots, n-1$ and $\left.\widetilde{L}_{n} y \in L^{p}\right\}$ by $\widetilde{L} y=\widetilde{L}_{n} y$.

TheOREm 3.2. For $1 \leqslant p<\infty \widetilde{L}$ on $L^{q}$ is the Banach space adjoint of $L$ on $L^{p}$; while for $p=\infty$ we define $L^{\prime}$ as an adjoint to $L$ on $L^{1}$ by $D\left(L^{\prime}\right)=$ $\left\{z \in L^{1} \mid\right.$ there is $z^{\prime} \in L^{1}$ with $\int_{-\infty}^{\infty} z L y d x=\int_{-\infty}^{\infty} z^{\prime} y d x$ for all $\left.y \in D_{L}\right\}$ and $L^{\prime} z=z^{\prime}$ for $z \in D\left(L^{\prime}\right)$, and again find $\widetilde{L}=L^{\prime}$.

Proof. In either case we must show that if $z \in L^{q}$ such that there is $z^{\prime} \in L^{q}$ with $\int_{-\infty}^{\infty} z L y d x=\int_{-\infty}^{\infty} z^{\prime} y d x$ for all $y \in D_{L, p}$ then $z \in D_{\tilde{L}, q}$ and $\tilde{L} z=z^{\prime}$. If $f=\left(L-\lambda_{0}\right) y$ then we may rewrite the above equation in the form

$$
\int_{-\infty}^{\infty} f(x)\left[z(x)-\int_{-\infty}^{\infty} G\left(\xi, x, \lambda_{0}\right)\left\{z^{\prime}(\xi)-\lambda_{0} z(\xi)\right\} d \xi\right] d x=0 .
$$

This holds for all $f$ in the range of $L-\lambda_{0}$, which is all of $L^{p}$ so

$$
z(x)=\int_{-\infty}^{\infty} G\left(\xi, x, \lambda_{0}\right)\left\{z^{\prime}(\xi)-\lambda_{0} z(\xi)\right\} d \xi .
$$

If $F$ is the column vector with $z^{\prime}-\lambda_{0} z$ in its first position and zeros elsewhere it is easy to see that $z$ is the $n$th entry in the column vector $Z(x)=i^{n} \int_{-\infty}^{\infty}$ $K\left(\xi, x, \lambda_{0}\right)^{\boldsymbol{T}} F(\xi) d \xi$. Using the definition of $K\left(x, \xi, \lambda_{0}\right)$ we see that $Z^{\prime}=-$ $\left(A^{T}+B^{T}\right) Z+i^{n} F$, and an examination of this set of equations yields the fact that $z \in D_{\tilde{L}, q}$ and $\tilde{L} z=z^{\prime}$.

Conversely, if $y \in D_{L, p}$ and $z \in D_{\tilde{L}, q}$ it follows easily that $\int z L y d x=\int y \widetilde{L} z d x$.

Corollary 3.1. For $1<p \leqslant \infty L$ on $L^{p}$ is the Banach space adjoint of $\tilde{L}$ on $L^{q}$. For $p=1 \tilde{L}^{\prime}=L$ where $\tilde{L}^{\prime}$ is defined as in the statement of the Theorem.

Proof. Except for the case $p=\infty$ this follows from Lemma 1.4 of Rota (7). For $p=\infty$ the graph of the Banach space adjoint of $L^{\prime}$ is the closure of the graph of $L$ in the $L^{1} \oplus L^{1}$ topology on $L^{\infty} \oplus L^{\infty}$. It is easy to see from the existence of a Green's function that the graph of $L$ is closed in this topology, so the result follows.

Thus the Banach space adjoint operator is closely related to the Lagrange adjoint differential operator (which does not exist in the normal sense unless $b_{j}(x) \in C^{n-j}$ ). For $p=2$ the usual Hilbert space adjoint of $L$ is given by $L^{*} y=\overline{\widetilde{L} \bar{y}}$ for $y \in D_{L, 2}$. In order to avoid making separate statements we shall define $L^{*}$ on $L^{p}$ by this equation for $y \in D_{\tilde{L}, p}=D_{L^{*}, p}$.

Since the solution of $\left(L^{*}-\bar{\lambda}\right) y=f$ will be given by the $n$th component of a solution to $Y^{\prime}=-\left(A^{*}+B^{*}\right) Y+(-i)^{n} F$ where $A^{*}$ and $B^{*}$ are the conjugate transposes of $A$ and $B$ respectively and $F$ is a column vector with $f$ in the first position and zero elsewhere, the work of $\$ \$ 1$ and 2 carries over almost completely. The polynomial $p(\mu)$ is replaced by $p^{*}(\mu)=\overline{p(\bar{\mu})}$. The quantities associated with the adjoint by this and subsequent work will be denoted by an asterisk superscript and to avoid confusion the conjugate transpose of a matrix $C$ will be denoted by $C^{(*)}$. 
Many results about the spectra of $L$ and $L^{*}$ are implicit above, but we shall gather them together here. We shall denote the resolvent set, spectrum, point spectrum, residual spectrum, and continuous spectrum of $L$ by $\rho(L), \sigma(L)$, $P_{\sigma}(L), R \sigma(L)$, and $C_{\sigma}(L)$ respectively.

Theorem 3.3. If $p(\mu)=\lambda$ has no real solutions $\lambda \in \rho(L)$ or $\lambda \in P \sigma(L)$ and if $\lambda$ is not a branch point then $\lambda \in P \sigma(L)$ if and only if $W_{j}(\lambda)=0$. The curve $\lambda=p(t)$ is contained in $\sigma(L)$, contains $C_{\sigma}(L)$ and $R \sigma(L)$, and the points of $P \sigma(L)$ and $R \sigma(L)$ lying on it form a nowhere dense set on any arc which does not lie between two $D_{j}^{\prime}$ 's in which the $W_{j}(\lambda)$ 's are identically zero. Each portion of $\sigma(L)$ is independent of $p$ except for the case $p=\infty$, where $\sigma(L)$ is all $P \sigma$ $(L)$ except perhaps for branch points lying on $\lambda=p(t)$.

Proof. If $p(\mu)=\lambda$ has no real solutions Theorem 2.1 shows that either $\lambda \in \rho(L)$ or $\phi_{1}, \phi_{2}, \ldots, \phi_{m}, \tilde{\phi}_{m+1}, \tilde{\phi}_{m+2}, \ldots, \tilde{\phi}_{n}$ are linearly dependent. In this case there are constants $c_{j}$, not all zero such that

$$
\psi \equiv \sum_{j=1}^{m} c_{j} \phi_{j}=\sum_{j=m+1}^{n} c_{j} \tilde{\phi}_{j}
$$

If $\chi$ is the first component of $\psi$ it is clear that $\chi \in L^{p}$ for any $p \geqslant 1$ and $(L-\lambda) \chi=0$, so $\lambda \in P \sigma(L)$.

On an arc of $\lambda=p(t)$ we have $W_{j}(\lambda)$ from one side and $W_{k}(\lambda)$ from the other side. If both are identically zero then the points of the arc are all in the closure of $P \sigma(L)$, and thus in $\sigma(L)$. If $W_{j}(\lambda)$ is not identically zero, then at any point on $\lambda=p(t)$ where it is not zero the only solution of $L y-\lambda y=f$ where $f(x)=0$ for $|x| \geqslant a$, which is in $L^{p}$, is the first component of $\int_{-a}^{a} K(x, \xi, \lambda)$ $F(\xi) d \xi$, where $F(\xi)$ is the column vector with $i^{n} f(\xi)$ as the last entry, and all others zero. If $(L-\lambda) y=0$ has a solution in $L^{p} \quad W_{j}(\lambda)$ must be zero as we shall see below so this is the only possibility. Now either $\phi_{m}(x) \backsim c e^{i t x}$ as $x \rightarrow \infty$ or $\tilde{\phi}_{m+1}(x) \backsim c e^{i t x}$ as $x \rightarrow-\infty$. Thus in order to have this solution belong to $L^{p}(P \neq \infty)$ we must have the $m$ th or the $(m+1)$ st entry in

$$
\int_{-a}^{a} \Psi_{j}^{-1}(\xi) F(\xi) d \xi
$$

equal to zero. It is easy to choose $F$ so that this is not true, so $(L-\lambda)^{-1}$ cannot everywhere be defined at such a point and $\lambda \in \sigma(L)$. As all other points on $\lambda=p(t)$ are in the closure of the points already mentioned we see that $\lambda=p(t)$ is contained in $\sigma(L)$.

In order to have $\lambda_{0}=p\left(t_{0}\right)$ belong to $P \sigma(L)$ for $p \neq \infty$ we must have a linear relation among the solutions which are exponentially small at $+\infty$ and those which are exponentially small at $-\infty$. This implies that $W_{j}(\lambda)$ and $W_{k}(\lambda)$ (coming from the two sides) must both be zero and thus such points cannot be dense on an arc of $\lambda=p(t)$ unless $W_{j}(\lambda)$ and $W_{k}(\lambda)$ are identically zero. The points of $R \sigma(L)$ cannot be dense on an arc unless those of $P \sigma\left(L^{*}\right)$ are dense on the corresponding arc of $\lambda=p^{*}(t)$, which means 
that $W^{*}{ }_{j}(\lambda)$ and $W^{*}{ }_{k}(\lambda)$ are identically zero. Thus the points off this arc of $\lambda=p(t)$ are all conjugates of points in $P \sigma\left(L^{*}\right)$, and so belong to $\sigma(L)$. By the above reasoning they belong to $P \sigma(L)$ and $W_{j}(\lambda)$ and $W_{k}(\lambda)$ must be identically zero. So $R \sigma(L)$ cannot be dense on an arc of $\lambda=p(t)$ which does not lie between two $D_{j}$ 's in which the $W_{j}(\lambda)$ 's are identically zero.

For $p=\infty$ we note that unless $\lambda$ is a branch point on $\lambda=p(t)$ there are $m$ solutions of $(L-\lambda) y=0$ which are bounded at $\infty$ and at least $n-m+1$ which are bounded at $-\infty$. As there must be a linear relation among $n+1$ solutions, we see that $\lambda \in P \sigma(L)$ for this case, and as the branch points on $\lambda=p(t)$ lie in the closure of these points they also lie in $\sigma(L)$.

In a consideration of $L^{*}$ we see that its properties can be developed from the system adjoint to (1.1). Thus as in Theorem 3.3 we see that if $p^{*}(\mu)=\lambda$ has no real solutions then $\lambda \in \rho\left(L^{*}\right)$ or $\lambda \in P \sigma\left(L^{*}\right)$. If $\lambda \in \rho\left(L^{*}\right)$ then $\bar{\lambda} \in \rho(L)$ and if $\bar{\lambda} \in P \sigma\left(L^{*}\right)$ then $\lambda \in \sigma(L)$. In the latter case as $p^{*}(\mu)=\lambda$ has no real solutions neither has $p(\mu)=\bar{\lambda}$ so $\bar{\lambda} \in \sigma(L)$ imples that $\bar{\lambda} \in P \sigma(L)$. Conversely we see that if $p(\mu)=\lambda$ has no real solutions and $\lambda \in P \sigma(L)$ then $\bar{\lambda} \epsilon P \sigma\left(L^{*}\right)$. Again from the proof of Theorem 3.3 applied to $L^{*}$ and the adjoint system we find that the curve $\lambda=p^{*}(t)$ splits up into $P \sigma\left(L^{*}\right), R \sigma$ $\left(L^{*}\right)$, and $C \sigma\left(L^{*}\right)$ as described there, and is contained in $\sigma\left(L^{*}\right)$. This proves

Corollary 3.2. If $\lambda$ does not lie on $\lambda=p^{*}(t)$ then $\lambda \in P \sigma\left(L^{*}\right)$ or $\rho\left(L^{*}\right)$ according as $\bar{\lambda} \in P \sigma(L)$ or $\rho(L)$. The curve $\lambda=p^{*}(t)$ splits up into $P \sigma\left(L^{*}\right)$, $R \sigma\left(L^{*}\right)$, and $C \sigma\left(L^{*}\right)$ as described in Theorem 3.3 , and is contained in $\sigma\left(L^{*}\right)$.

4. The spectral resolution of $L$ and $L^{*}$. For certain special cases we shall obtain a type of spectral resolution. We first obtain an expansion of the Green's function in terms of a sum of eigenfunctions and an integral involving improper eigenfunctions. From this we can develop an expansion for a suitably restricted class of functions, prove an analozue of the Parseval equality, and define for exch boxnded Borel set $M$ a closed, densely defined operator $E(M)$ which com nutes with $L$ and has the properties: $E(M) E(N)=E(M \cap N)$ and $E(M)+E(N)=E(M \cup N)$ if $M \cap N=\phi$. In special cases these projections may all be bounded, or even uniformly bounded, in which case $L$ is an unbounded spectral operator. We shall now proceed to develop the expansion of the Green's function for the special cases under consideration.

We shall assume that $P \sigma(L)$ and $P \sigma\left(L^{*}\right)$ are finite and do not intersect $\lambda=p(t)$ and $\lambda=p^{*}(t)$ respectively. We shall also assume that on $\lambda=p(t)$ and $\lambda=p^{*}(t)$ the functions $W_{j}(\lambda)$ and $W^{*}{ }_{j}(\lambda)$ which are defined, are not zero.

This results in considerable simplification, and it is quite possible that the method may be generalized to deal with the less special case where the only additional assumption is that $P \sigma(L)$ and $P \sigma\left(L^{*}\right)$ are finite. Without this assumption, however, the convergence difficulties seem to be quite formidable.

We shall consider the contour integral

$$
\frac{1}{2 \pi i} \oint_{C_{\delta, R}} \frac{G(x, \xi, \mu)}{\mu-\lambda} d \mu=I(\delta, R),
$$


where $C_{\delta, R}$ is a compound contour which we shall now describe. Let $H_{\delta}^{\prime}$ consist of all points $\lambda$ which are within a distance $\delta$ of $\lambda=p(t)$. This set has a boundary consisting of piecewise differentiable curves. Let $H_{\delta, R}=\{\lambda|| \lambda \mid$ $\left.\leqslant R, \lambda \notin H_{\delta}^{\prime}\right\}$, and let $C_{\delta, R}$ be the boundary of $H_{\delta, R}$, traversed in the positive sense. We assume $\delta$ is so small and $R$ so large that $P \sigma(L) \subset H_{\delta, R}$ and so that for each $D_{j}, D_{j} \cap H_{\delta, R}$ is non-empty.

The evaluation of $I(\delta, R)$ will be performed in two ways: by residues, and by direct integration for the case $\delta \rightarrow 0, R \rightarrow \infty$. We shall first proceed with the residue technique. The singularities of the integrand occur at $\mu=\lambda$, and at $\mu=\lambda_{1}, \lambda_{2}, \ldots, \lambda_{p}$ where $\lambda_{1}, \lambda_{2}, \ldots, \lambda_{p}$ constitute $P \sigma(L)$. We note that $C_{\delta, R}$ consists of loops, one in each $D_{j}$, and each of the singularities is enclosed in one of these loops. Thus, by residues, $I(\delta, R)$ is simply the sum of the residues of $G(x, \xi, \mu) /(\mu-\lambda)$ at each of these singularities.

Lemma 4.1. The residue at $\mu=\lambda$ is $G(x, \xi, \lambda)$ and that at $\lambda_{j}$ is minus the singular portion of the Laurent expansion of $G(x, \xi, \lambda)$ about $\lambda_{j}$.

Proof. The first statement is obvious, and the second is almost so. Since the singularity of $G(x, \xi, \lambda)$ at $\lambda_{j}$ arises from a zero of the appropriate $W_{k}(\lambda)$, it must be a pole. Suppose that this pole is of order $r_{j}$ and that the singular part of the Laurent expansion is

$$
\sum_{\alpha=1}^{r_{j}} G_{\alpha}^{(j)}(x, \xi)\left(\lambda-\lambda_{j}\right)^{-\alpha}
$$

Then in the neighbourhood of $\lambda_{j}$ the integrand of $I(\delta, R)$ is equal to

$$
\begin{aligned}
-\frac{1}{\left(\lambda-\lambda_{j}\right)}\left(1-\frac{\mu-\lambda_{j}}{\lambda-\lambda_{j}}\right)^{-1} \sum_{\alpha=1}^{r} G_{\alpha}^{(j)}(x, \xi)\left(\lambda-\lambda_{j}\right)^{-\alpha} & \\
= & -\frac{1}{\left(\lambda-\lambda_{j}\right)} \sum_{m=1}^{r_{j}}\left(\mu-\lambda_{j}\right)^{-m} \sum_{\alpha=0}^{r_{j}-m}\left(\lambda-\lambda_{j}\right)^{-\alpha} G_{m+\alpha}^{(j)}(x, \xi) \\
& + \text { terms in higher powers of }\left(\mu-\lambda_{j}\right) .
\end{aligned}
$$

Thus the residue is obviously

$$
-\sum_{\alpha=0}^{r_{j}-1}\left(\lambda-\lambda_{j}\right)^{-\alpha-1} G_{\alpha+1}^{(j)}(x, \xi)=-\sum_{\alpha=1}^{r_{j}}(\lambda-\lambda)^{-\alpha} G_{\alpha}^{(j)}(x, \xi) .
$$

In order to resolve the $G_{\alpha}^{(j)}(x, \xi)$ 's in terms of the characteristic and associated functions we shall need to know some of their properties.

LEMMA 4.2. There is a positive number $u_{j}$ such that

$$
\left|G_{\alpha}^{(j)}(x, \xi)\right| \leqslant K e^{-u_{j}|x-\xi|}, \alpha=1,2, \ldots r_{j} .
$$

For fixed $\xi G_{\alpha}^{(j)}(x, \xi)$ belongs to the domain of $L$ and

$$
\left(L-\lambda_{j}\right) G_{\alpha}^{(j)}(x, \xi)= \begin{cases}0 & \alpha=r_{j} \\ G_{\alpha+1}^{(j)}(x, \xi) & \alpha=1,2, \ldots, r_{\jmath}-1 .\end{cases}
$$


For fixed $x, \overline{G_{\alpha}^{(j)}(x, \xi)}=G_{\alpha^{(j)}}(\xi, x)$ belongs to the domain of $L^{*}$ and

$$
\left(L^{*}-\bar{\lambda}_{\jmath}\right) \overline{G_{\alpha}^{(j)}(x, \xi)}= \begin{cases}\frac{0}{G_{\alpha+1}^{(\bar{j})}(x, \xi)} & \alpha=r_{j} \\ & \alpha=1,2, \ldots, r_{j}-1 .\end{cases}
$$

Proof. If we note that for each $\lambda_{j}$ there is a circle $C_{j}$ surrounding it and no other characteristic values, which lies completely in $H_{\delta, R}$; we see that

$$
G_{\alpha}^{(j)}(x, \xi)=\frac{1}{2 \pi i} \oint_{c_{j}}\left(\lambda-\lambda_{j}\right)^{\alpha-1} G(x, \xi, \lambda) d \lambda .
$$

We have noted that for points such as $\lambda$ on $C_{\text {, }}$

$$
|G(x, \xi, \lambda)| \leqslant K \exp [-u|x-\xi|]
$$

where $u$ is less than the minimum absolute value of the imaginary parts of the solutions of $p(\mu)=\lambda$. As these must have a positive minimum on $C_{j}$ we take $u_{j}$ equal to one-half of that and $|G(x, \xi, \lambda)| \leqslant K \exp \left[-u_{j}|x-\xi|\right]$ for all $\lambda$ on $C_{j}$. Thus if the radius of $C_{j}$ is $r_{j}$ we have

$$
\begin{aligned}
\left|G_{\alpha}^{(j)}(x, \xi)\right| & \leqslant \frac{1}{2 \pi} \int_{0}^{2 \pi} r_{j}^{\alpha-1} K \exp \left[-u_{j}|x-\xi|\right] r d \theta \\
& =K r_{j}^{\alpha} \exp \left[-u_{j}|x-\xi|\right],
\end{aligned}
$$

which proves (4.2).

If $\Gamma_{0}(x, \xi, \lambda)$ is defined to be the entry in the upper right-hand corner of the matrix

$$
\Gamma(x, \xi, \lambda)= \begin{cases}0 & \xi \geqslant x \\ (-i)^{n} M e^{i \theta(x-\xi)} M^{-1} & x \leqslant \xi\end{cases}
$$

then $\Gamma_{0}$ has the same discontinuity at $x=\xi$ as $G(x, \xi, \lambda)$, and is analytic in a neighbourhood of $\lambda_{j}$ which includes $C_{j}$. Thus $G(x, \xi, \lambda)-\Gamma_{0}(x, \xi, \lambda)$ belongs to $C^{n}$ and so must

$$
G_{a}^{(j)}(x, \xi)=\frac{1}{2 \pi i} \oint_{C_{j}}\left(\lambda-\lambda_{j}\right)^{\alpha-1}\left[G(x, \xi, \lambda)-\Gamma_{0}(x, \xi, \lambda)\right] d \lambda .
$$

From this we easily obtain (4.3), and (4.4) follows in a similar manner.

In order to obtain an expression for $G_{\alpha}{ }^{(j)}(x, \xi)$ we consider the $L^{2}$ case separately. It is known that in the neighbourhood of an isolated pole $\lambda_{j}$ of the resolvent $R_{\lambda}=(L-\lambda I)^{-1}$ can be expanded in the form

$$
R_{\lambda}=-\frac{Q_{j}^{r_{j}-1}}{\left(\lambda-\lambda_{j}\right)^{r_{j}}}-\frac{Q_{j}^{r_{j}-2}}{\left(\lambda-\lambda_{j}\right)^{r_{j}-1}}-\ldots-\frac{Q_{j}}{\left(\lambda-\lambda_{j}\right)^{2}}-\frac{P_{j}}{\lambda-\lambda_{j}}+\ldots
$$

where $\left(L-\lambda_{j}\right) P_{j}=P_{j}\left(L-\lambda_{j}\right)=Q_{j}, P_{j}^{2}=P_{j}, P_{j} Q_{j}=Q_{j} P_{j}=Q_{j}$ and $Q_{j}{ }^{r_{j}}=0$. Let $P_{j 0}$ be the orthogonal projection on the orthogonal complement of $\left(I-P_{j}\right) \mathfrak{S}$ (where $\left.\mathfrak{S}=L^{2}\right)$. Now if $P_{j 0} z=z$ and $P_{j} z=0$ then $z \in$ $\left(I-P_{j}\right) \mathfrak{S}$ and so $z \in\left(I-P_{j 0}\right) \mathfrak{S}$, which implies that $z=0$. Since $\left(I-P_{j}\right)\left(I-P_{j 0}\right) z=\left(I-P_{j 0}\right) z$ for any $z \in \mathfrak{S}$ we have 


$$
\begin{aligned}
z & =P_{j 0} z+\left(I-P_{j 0}\right) z \\
& =P_{j}\left(P_{j 0} z\right)+\left(I-P_{j}\right)\left[P_{j 0} z+\left(I-P_{j 0}\right) z\right] \\
& =P_{j} z+\left(I-P_{j}\right) z
\end{aligned}
$$

for any $z \in \mathfrak{S}$. These two latter expressions both give $z$ as the sum of a vector in $P_{j} \mathfrak{S}$ plus one in $\left(I-P_{j}\right) \mathfrak{S}$, and as this is unique $P_{j}\left(P_{j 0} z\right)=P_{j} z$. Similarly $P_{j 0}\left(P_{j} z\right)=P_{j 0} z$ for all $z \in \mathfrak{S}$ and so $P_{j}$ maps $P_{j 0} \mathfrak{S}$ one to one onto $P_{j} \mathfrak{S}$, and its inverse is $P_{j 0}$. If $\left\{e_{k}\right\}$ is an orthonormal basis of $P_{j 0} \mathfrak{S}$ then for any $z \in \mathfrak{S}$

$$
P_{j} z=P_{j} P_{j 0} z=P_{j} \sum_{k}\left(z, e_{k}\right) e_{k}=\sum_{k}\left(z, e_{k}\right) P_{j} e_{k},
$$

from which it follows that

$$
P_{j z}^{*}=\sum_{k}\left(z, P e_{k}\right) e_{k}
$$

and $\left(P e_{k}, e_{1}\right)=\delta_{k 1}$. Thus $P_{j 0} \mathfrak{F}=P^{*}{ }_{j} \mathfrak{S}$ can be identified with the dual space of $P_{j} \mathfrak{S}$. Since every vector $z \in P_{j} \mathfrak{W}$ which satisfies $Q_{j} z=0$ is a characteristic function of $L$ corresponding to the characteristic value $\lambda_{j}$, the dimension of the null-space of $Q_{j}$ restricted to $P_{j} \mathfrak{S}$ must be finite $(\leqslant n / 2)$. Using this and the fact that $Q_{j}{ }^{{ }_{j}}=0$, a simple induction shows that $\operatorname{dim} P_{j} \mathfrak{S}$ is less than or equal to $\left(r_{j}-1\right)^{\frac{1}{2} n}$. Thus we may now choose a basis of $P_{j} \mathfrak{S}$ consisting of

$$
\begin{gathered}
x_{j 1}, Q_{j} x_{j 1}, \ldots, Q_{j}^{s j 1} x_{j 1} ; \\
x_{j 2}, Q_{j} x_{j 2}, \ldots, Q_{j}^{s j 2} x_{j 2} ; \\
\ldots \\
x_{j p_{j}}, Q_{j} x_{j p_{j}}, \ldots, Q_{j}^{s j p_{j}} x_{j p_{j}} ;
\end{gathered}
$$

where

$$
r_{j}-1=s_{j 1} \geqslant s_{j 2} \geqslant \ldots \geqslant s_{j p_{j}} .
$$

It is easy to see that the dual basis for $P^{*}{ }_{j} \mathfrak{T}$ may be written in the form

$$
\begin{aligned}
& x_{j 1}^{*}, Q_{j}^{*} x_{j 1}^{*}, \ldots, Q_{j}^{*_{s j 1}} x_{j 1}^{*} ; \\
& x_{j 2}^{*}, Q_{j}^{*} x_{j 2}^{*}, \ldots, Q_{j}^{*_{s 2}} x_{j 2}^{*} ; \\
& x_{j p_{j}}^{*}, Q_{j}^{*} x_{j p_{j}}^{*}, \ldots, Q_{j}^{*_{s j p_{j}}} x_{j p_{j}}^{*} ;
\end{aligned}
$$

where $\left(Q_{j}{ }^{i} x_{j k}, Q^{*}{ }_{j} x^{*}{ }_{j m}\right)=\delta_{i+l, s_{j k}} \delta_{k m}$. Thus

$$
\begin{aligned}
Q_{j}^{l} x & =Q_{j}^{l} P_{j} x=Q_{j}^{l} \sum_{\alpha=1}^{p_{j}} \sum_{\beta=0}^{s_{j} \alpha}\left(x, Q_{j}^{* s_{j \alpha}-\beta} x_{j \alpha}^{*}\right) Q_{j}^{\beta} x_{j \alpha} \\
& =\sum_{\alpha=1}^{p_{j}} \sum_{\beta=0}^{s_{j \alpha-l}}\left(x, Q_{j}^{*_{s j \alpha-\beta}} x_{j \alpha}^{*}\right) Q_{j}^{\beta+l} x_{j \alpha} .
\end{aligned}
$$

Now each of these basis elements is a function, and we see that $P_{j}$ and $Q_{j}{ }^{l}\left(l=1,2, \ldots, r_{j}-1\right)$ are all integral operators, with kernels $-G_{1}{ }^{(j)}$ $(x, \xi)$ and $-G_{l+1}{ }^{(j)}(x, \xi)$ respectively. This yields expressions for $G_{\alpha}{ }^{(j)}(x, \xi)$ 
for which we shall make some changes in notation. Let $\chi_{k l}^{(j)}(x)$ be the function represented by $Q_{j}{ }^{s}{ }^{-l} x_{j k}$ and $\chi_{k l}^{*}{ }^{(j)}(x)$ be the function represented by $Q^{*}{ }_{j}{ }_{j k}{ }^{-l} x^{*}{ }_{j k}$. Then we may summarize the preceding results in the following theorem.

\section{THEOREM 4.1. Using residue methods we obtain}

$$
I_{\delta, R}=G(x, \xi, \lambda)-\sum_{j=1}^{p} \sum_{\alpha=1}^{\tau_{j}} G_{\alpha}^{(j)}(x, \xi)\left(\lambda-\lambda_{j}\right)^{-\alpha} .
$$

For each $j(j=1,2, \ldots, p)$ there are numbers $q_{j}$ and

$$
s_{j q_{j}} \leqslant s_{j q_{j}-1} \ldots s_{j 1}=r_{j}-1
$$

and functions $\chi_{k l}{ }^{(j)}(x), \chi_{k l}^{*}{ }^{(j)}(x)\left(k=1, \ldots, q_{j}, l=0,1,2, \ldots, s_{j k}\right)$ such that $\chi_{k l}^{(j)}(x) \in D_{L, p}, \chi_{k l}^{*}{ }^{(j)}(x) \in D_{L *, p}$ for all $p$,

$$
\begin{aligned}
& \left|\chi_{k l}^{(j)}(x)\right| \leqslant K e^{-u_{j}|x|},\left|\chi_{k l}^{*(j)}(x)\right| \leqslant K e^{-u_{j}|x|} ; \\
& \left(L-\lambda_{j}\right) \chi_{k l}^{(j)}= \begin{cases}0 & l=0 \\
\chi_{k l-1}^{(j)} & l=1,2, \ldots, s_{j k},\end{cases} \\
& \left(L^{*}-\bar{\lambda}_{j}\right) \chi_{k l}^{*(j)}= \begin{cases}0 & l=0 \\
\chi_{k l-1}^{*}(j) & l=1,2, \ldots, s_{j k},\end{cases} \\
& \int_{-\infty}^{\infty} \chi_{k l}^{(j)}(x) \chi_{k^{\prime} l^{\prime}}^{*\left(j^{\prime}\right)}(x) d x=\delta_{j j^{\prime}} \delta_{k k^{\prime}} \delta_{l+l^{\prime}, s_{j k} .} .
\end{aligned}
$$

Also

$$
G_{l}^{(j)}(x, \xi)=-\sum_{\alpha=1}^{p_{j}} \sum_{\beta=0}^{s_{j \alpha}-l} \chi_{\alpha \beta}^{(j)}(x) \overline{\chi_{\alpha, s_{j \alpha}-l-\beta}^{*}(\bar{j})}(\xi),
$$

where a sum from zero to a negative integer is zero.

Proof. Relation (4.5) follows from Lemma 4.1. The estimates of the $\chi^{(j)}$ 's and $\chi^{*(j)}$ 's follow from (4.2) and their linear independence, while the other formulae follow from the construction of the $\chi^{(j)}$ 's and $\chi^{*(j)}$ 's, and from well-known properties of characteristic and associated functions.

We must now proceed to the evaluation of $I_{\delta, R}$ directly. From Theorem 4.1 $I_{\delta, R}$ is independent of $\delta$ and $R$, and we wish the limit of the direct evaluation in terms of line integrals as $\delta \rightarrow 0$ and $R \rightarrow \infty$. We shall denote the portion of $I_{\delta, R}$ which arises from the integral over arcs of the circle $|\lambda|=R$ by

$$
\int_{|\lambda|=R} G(x, \xi, \mu) /(\mu-\lambda) d \mu
$$

LEMMA 4.3 .

$$
\lim _{R \rightarrow \infty} \int_{|\lambda|=R} \frac{G(x, \xi, \mu)}{\mu-\lambda} d \mu=0,
$$

uniformly in $\delta$ for $\delta \geqslant 0$, provided $x \neq \xi$. 
Proof. As

$$
\mu_{k}=\lambda^{1 / n} \exp \left[\frac{2 \pi k i}{n}\right]\left(1+O\left(\lambda^{-1 / n}\right)\right)
$$

for large $|\lambda|$ we see that the function $u(\lambda)$ in $|G(x, \xi, \lambda)| \leqslant K \exp [-u(\lambda)$ $\left.\left|x_{-}-\xi\right|\right]$ approaches zero as $R e^{i \theta}$ approaches $R e^{i \theta_{0}}=p\left(t_{0}\right)$ in the same manner as

$$
R^{1 / n} \sin \frac{\theta-\theta_{0}}{n}
$$

Thus we may divide one of these arcs of $|\lambda|=R$ (from $\theta_{1}$ to $\theta_{2}$ say) by $\theta_{1}{ }^{\prime}$ and $\theta_{2}{ }^{\prime}$ such that

$$
\left|G\left(x, \xi, R e^{i \theta}\right)\right| \leqslant\left\{\begin{array}{l}
K \exp \left[-c R^{1 / n} \sin \left[\frac{\theta-\theta_{1}}{n}\right]|x-\xi|\right] \theta_{1} \leqslant \theta \leqslant \theta_{1}^{\prime} \\
K \exp \left[-c R^{1 / n}|x-\xi|\right] \\
K \exp \left[-c R^{1 / n} \sin \left[\frac{\theta_{2}-\theta}{n}\right]|x-\xi|\right] \theta_{2}^{\prime} \leqslant \theta \leqslant \theta_{2}^{\prime}
\end{array}\right.
$$

As there are two of these arcs we have

$$
\begin{aligned}
&\left|\int_{|\lambda|=R} \frac{G(x, \xi, \mu)}{\mu-\lambda} d \mu\right| \\
& \leqslant 2 K \int_{\theta_{1}}^{\theta_{1^{\prime}}} \frac{\exp \left[-c R^{1 / n} \sin \frac{\theta-\theta_{1}}{n}|x-\xi|\right]}{R-|\lambda|} R d \theta \\
&+ 2 K \int_{\theta_{1^{\prime}}}^{\theta_{2^{\prime}}} \frac{\exp \left[-c R^{1 / n}|x-\xi|\right]}{R-|\lambda|} R d \theta \\
&+ 2 K \int_{\theta_{2^{\prime}}}^{\theta_{2^{\prime}}} \frac{\exp \left[-c R^{1 / n} \sin \frac{\theta_{2}-\theta}{n}|x-\xi|\right]}{R-|\lambda|} R d \theta \\
& \leqslant K \int_{0}^{\pi / 2} \exp \left[-c R^{1 / n} \sin \phi|x-\xi|\right] d \phi \\
& \leqslant K \int_{0}^{\pi / 2} \exp \left[-\frac{2 c R^{1 / n} \phi}{\pi}|x-\xi|\right] d \phi \\
& \leqslant \frac{K \pi}{2 c R^{1 / n}} \frac{\exp -\xi \mid}{\mid x-}
\end{aligned}
$$

where $K$ has been increased as necessary.

Thus in the limit we need only consider the integrals along the boundary of $H_{\delta, R}$ as $R \rightarrow \infty$ and $\delta \rightarrow 0$. The arcs have the form

$$
\lambda=p(t) \pm \frac{i \delta p^{\prime}(t)}{\left|p^{\prime}(t)\right|}
$$


and so may be written in the limit as $\delta \rightarrow 0$

$$
\int_{\mu=p(t)} \frac{G(x, \xi, \mu+)-G(x, \xi, \mu-)}{\mu-\lambda} d \mu
$$

where $G(x, \xi, \mu+)$ is the limit of the Green's function from one side, and $G(x, \xi, \mu-)$ is the limit from the other side. Our assumption that $W(\lambda)$ has no zeros in $\lambda=p(t)$ implies that these limits exist and are continuous, so the limit of the integral exists as long as $R$ is finite. We must now evaluate $G(x, \xi, \mu+)-G(x, \xi, \mu-)$ to show that it exists when $R=\infty$ as well. First note that they are uniformly bounded in $x, \xi$ and $\mu$, and have the same discontinuity in the $(n-1)$ st derivative at $x=\xi$. This implies that as functions of $x$ they belong to $C^{n}$ and satisfy $(L-\mu) y=0$. It is easy to see that this equation has precisely as many linearly independent uniformly bounded solutions as $p(t)=\mu$ has real solutions (unless $\mu$ is an eigenvalue, which we have excluded). Let this number be $\sigma(\mu)$, where $\mu=p(t)$, and denote the linearly independent bounded solutions by $\chi_{1}(x, \mu), \chi_{2}(x, \mu), \ldots$, $\chi_{\sigma(\mu)}(x, \mu)$. Then it is obvious that

$$
G(x, \xi, \mu+)-G(x, \xi, \mu-)=2 \pi i \sum_{j=1}^{\sigma(\mu)} \chi_{j}(x, \mu) \overline{\chi_{j}^{*}(\xi, \bar{\mu})} .
$$

We see also that $\left(L^{*}-\mu\right) \chi_{j}^{*}(x, \mu)=0$ and that $\chi^{*}{ }_{j}(x, \mu)$ is bounded. In fact, if we investigate the asymptotic behaviour of $G(x, \xi, \mu+)-G(x, \xi, \mu-)$ for large values of $\mu$ we find that it behaves like

$$
\sum_{j=1}^{\sigma(\mu)} \frac{i e^{i \nu_{j}(x-\xi)}}{p^{\prime}\left(\nu_{j}\right)}
$$

where $\sigma(\mu)$ is 1 or 2 and $\nu_{1}$ (and $\nu_{2}$ ) are the real solutions of $p(\nu)=\mu$. Thus the integral over the portion of $\lambda=p(t)$ which extends to infinity will be less than or equal to

$$
K \int_{t_{0}}^{\infty} \frac{d t}{|p(t)-\lambda|}
$$

which clearly exists for $n \geqslant 2$.

THEOREM 4.2 .

$$
\begin{aligned}
G(x, \xi, \lambda)=\sum_{j=1}^{p} \sum_{\alpha=1}^{r_{j}} G_{\alpha}^{(j)}(x, \xi) & \left(\lambda-\lambda_{j}\right)^{-\alpha} \\
& +\int_{\mu=p(t)} \sum_{j=1}^{\sigma(\mu)} \frac{\chi_{j}(x, \mu) \overline{\chi_{j}^{*}(\xi, \bar{\mu})}}{\mu-\lambda} d \mu .
\end{aligned}
$$

Proof. This follows immediately from the evaluation of $I_{\delta, R}$ as $\delta \rightarrow 0$ and $R \rightarrow \infty$ above, and from the previous evaluation by residues in (4.5).

We may immediately obtain a corresponding expansion of $G^{*}(x, \xi, \lambda)$, and also the following expansion of a certain class of functions.

Theorem 4.3. If $f \in D_{L, 1}, g \in D_{L^{*}, 1}$ then 


$$
\hat{f}_{j}(\lambda)=\int_{-\infty}^{\infty} f(x) \overline{\chi_{j}^{*}} \overline{(x, \bar{\lambda})} d x \text { and } \hat{g}_{j}^{*}(\lambda)=\int_{-\infty}^{\infty} g(x) \overline{\chi_{j}(x, \overline{\bar{\lambda}})} d x
$$

exist and are integrable along $\lambda=p(t)$ and $\lambda=p^{*}(t)$ respectively. Also

$$
\begin{aligned}
f(x)=\sum_{j=1}^{p} \sum_{\alpha=1}^{q_{j}} \sum_{\beta=0}^{s j \alpha} \chi_{\alpha \beta}^{(j)}(x) & \left(f, \chi_{\alpha, s_{j}-\beta}^{*(j)}\right) \\
& +\int_{\lambda=p(t)} \sum_{j=1}^{\sigma(\lambda)} \chi_{j}(x, \lambda) \hat{f}_{j}(\lambda) d \lambda, \\
g(x)=\sum_{j=1}^{p} \sum_{\alpha=1}^{q_{j}} \sum_{\beta=0}^{s_{j \alpha}} \chi_{\alpha \beta}^{*(j)}(x)\left(g, \chi_{\alpha, s j \alpha-\beta}^{(j)}\right) & +\int_{\lambda=p^{*}(t)} \sum_{j=1}^{\sigma(\lambda)} \chi_{j}^{*}(x, \lambda) \hat{g}_{j}^{*}(\lambda) d \lambda .
\end{aligned}
$$

There is also an analogue of the Parseval equality:

$$
\begin{aligned}
(f, g)=\sum_{j=1}^{p} \sum_{\alpha=1}^{q_{j}} \sum_{\beta=0}^{s_{j \alpha}}\left(f, \chi_{\alpha, s_{j_{\alpha}}-\beta}^{*}\right)\left(\chi_{\alpha \beta}, g\right) & \\
& +\int_{\lambda=p(t)} \sum_{j=1}^{\sigma(\lambda)} \hat{f}_{j}(\lambda) \overline{\hat{g}}_{j}^{*} \overline{(\lambda)} d \lambda,
\end{aligned}
$$

and one may obtain a slightly different expression by interchanging $f$ and $g$, taking conjugates, and changing the variable in the integral to $\lambda=p^{*}(t)$.

Proof. The existence of $\hat{f}_{j}^{*}(\lambda)$ and $\hat{g}_{j}^{*}(\lambda)$ is obvious, and the expansions (4.7) follow from the identities

$$
f=(L-\lambda) \int_{-\infty}^{\infty} G(x, \xi, \lambda) f(\xi) d \xi, g=\left(L^{*}-\lambda\right) \int_{-\infty}^{\infty} G^{*}(x, \xi, \lambda) g(\xi) d \xi
$$

exactly as in (4). Relation (4.8) follows when we note that $f \in D_{L, 1}$ implies $f \in L^{2}$ as well.

Formally we define the spectral resolution $E(M)$ in terms of the kernel

$$
E(x, \xi ; M)=-\sum_{\lambda_{j} \in M} G_{1}^{(j)}(x, \xi)+\int_{p(t) \cap_{M}} \sum_{j=1}^{\sigma(\mu)} \chi_{j}(x, \mu) \overline{\chi_{j}^{*} \overline{(\xi, \bar{\mu})}} d \mu .
$$

It is easy to see that this exists for any bounded Borel set $M$, and that for any $f \in D_{L, p} E(M) f=\int_{-\infty}^{\infty} E(x, \xi ; M) f(\xi) d \xi$ exists. Thus for any bounded Borel set $E(M)$ is a closed, densely defined operator. Also, for any such $f, E(M) f \in D_{L, p}$, and if $E(M) L f$ exists it is equal to $L E(M) f$, so $L$ and $E(M)$ commute in a certain sense. It is also clear that if $M \cap N=\phi$, then $E(M \cup N)$ $=E(M)+E(N)$. It is not quite so clear that $E(M) E(N)=E(M \cap N)$, but if we note that for $\lambda=p(t), \chi_{m}(x, \lambda) \in D_{L, \infty}$, we see that $\chi_{m}(x, \lambda)=$ $\left(\lambda-\lambda_{0}\right) \int_{-\infty}^{\infty} G\left(x, \xi, \lambda_{0}\right) \chi_{m}(\xi, \lambda) d \xi$. It is easy to see that $\int_{-\infty}^{\infty} G^{(j)}(x, \xi) \chi_{m}$ $(\xi, \lambda) d \xi=0$ so that

$$
\chi_{m}(x, \lambda)=\left(\lambda-\lambda_{0}\right) \int_{-\infty}^{\infty} \int_{\mu=p(t)}^{-\infty} \sum_{j=1}^{\sigma(\mu)} \frac{\chi_{j}(x, \mu) \overline{\chi_{j}^{*}(\xi, \bar{\mu})}}{\mu-\lambda_{0}} \chi_{m}(\xi, \lambda) d \mu d \xi,
$$


and for $g \in D_{L^{*}, 1}$

$$
\begin{aligned}
& \overline{\check{g}_{m}^{*}(\bar{\lambda})}=\int_{-\infty}^{\infty} \int_{\mu=p(t)} \sum_{j=1}^{\sigma(\mu)} \frac{\lambda-\lambda_{0}}{\mu-\lambda_{0}} \overline{\hat{g}_{j}^{*}(\bar{\mu})} \chi_{m}(\xi, \lambda) \overline{\chi_{j}^{*}(\xi, \bar{\mu})} d \mu d \xi \\
& =\lim _{R_{1}, R_{2} \rightarrow \infty} \int_{\mu=p(t)} \frac{\lambda-\lambda_{0}}{\mu-\lambda_{0}} \sum_{j=1}^{\sigma(\mu)} \overline{\hat{g}_{j}^{*}} \overline{(\bar{\mu})} \int_{-R_{1}}^{R_{2}} \chi_{m}(\xi, \lambda) \overline{\chi_{j}^{*}(\xi, \bar{\mu})} d \xi d \mu .
\end{aligned}
$$

Thus we see that the distribution $\int_{\infty}^{\infty} \chi_{m}(\xi, \lambda) \chi^{*}{ }_{j}(\xi, \bar{\mu}) d \xi$ is zero unless $j=m$ in which case it is $\delta(\mu-\lambda)$. Thus the kernel $\int_{-\infty}^{\infty} E(x, z ; M) E(z, \xi, N) d z$ of $E(M) E(N)$ is easily shown to be equal to $E(x, \xi ; M \cap N)$, and we have $E(M) E(N)=E(M \cap N)$.

In some cases this resolution will consist of bounded projections, and may even be uniformly bounded. In this case it will be a spectral measure in the sense of Dunford (3) if it is also countably additive. However, this will not be true in general, for if the perturbing operator $L_{1}$ is absent $E(M)$ is a simple function of the operator with kernel $\frac{1}{2} \int_{M_{\Omega} R} \exp [i \sigma(x-\xi)] d \sigma$. This gives a self-adjoint spectral measure on $L^{2}$, but Rota (7) has pointed out that it is unbounded on any other $L^{p}$ space.

\section{REFERENCES}

1. M. S. Brodskiĭ and M. S. Livšic, Spectral analysis of non-self-adjoint operators and intermediate systems, Usp. Mat. Nauk. (N. S.), 18, no. 1 (79) (1958), 3-85.

2. E. A. Coddington and N. Levinson, Theory of ordinary differential equations (New York, 1955).

3. N. Dunford, Spectral operators, Pac. J. Math., 4 (1954), 321-354.

4. R. R. D. Kemp, A singular boundary value problem for a non-self-adjoint differential operator, Can. J. Math., 10 (1958), 447-462.

5 M. S. Livšic, On spectral decomposition of linear non-self-adjoint operators, Amer. Math. Soc. Translations (2), 5 (1957), 67-114.

6. M. A. Naimark, Investigation of the spectrum and expansion in eigenfunctions of a non-selfadjoint differential operator of the second order on a semi-axis, Trudy Moskov Mat. Obsc, 3 (1954), 181-270.

7. G. C. Rota, Extension theory of differential operators I, Comm. Pure and App. Math., 11 (1958), 23-66.

8. J. Schwartz, Perturbations of spectral operators, and applications I, bounded perturbations, Pac. J. Math., 4 (1954), 415-458.

Queen's University 